\title{
Surgical management of fibrotic encapsulation of the fluocinolone acetonide implant in CAPN5- associated proliferative vitreoretinopathy
}

This article was published in the following Dove Press journal:

Clinical Ophthalmology

7 June 2013

Number of times this article has been viewed

\author{
Paul S Tlucek' \\ James C Folk' \\ Warren $\mathrm{M} \mathrm{Sobol}^{2}$ \\ Vinit B Mahajan ${ }^{1,3}$ \\ 'Department of Ophthalmology and \\ Visual Sciences, University of lowa, \\ lowa City, IA, USA; ${ }^{2}$ Retina Physicians \\ and Surgeons, Dayton, OH, USA; \\ ${ }^{3}$ Omics Laboratory, University of \\ lowa, lowa City, IA, USA
}

Objective: To review fibrosis of fluocinolone acetonide (FA) implants in subjects with CAPN5 autosomal dominant neovascular inflammatory vitreoretinopathy (ADNIV).

Methods: A retrospective case series was assembled from ADNIV patients in which there was fibrotic encapsulation of a fluocinolone acetonide implant. CAPN5 genotypes and surgical repair techniques were reviewed.

Results: Two eyes of two ADNIV patients developed a fibrotic capsule over the fluocinolone acetonide implant. Both patients had Stage IV disease. Patient A had a c.731T $>$ C mutation in the CAPN5 gene and patient $\mathrm{B}$ had a c.728G $>\mathrm{T}$ mutation. The fibrotic membrane was surgically excised and the implant function was restored.

Conclusion: The exuberant fibrotic response in later stages of ADNIV may be resistant to local immunosuppression with steroids. Surgical excision of fibrotic membranes over FA implants can reestablish local steroid delivery in cases of severe proliferative vitreoretinopathy.

Keywords: autosomal dominant neovascular inflammatory vitreoretinopathy, ADNIV, CAPN5, calpain-5, Retisert, fluocinolone acetonide, fibrotic encapsulation

\section{Introduction}

Autosomal dominant neovascular inflammatory vitreoretinopathy (ADNIV) is an inherited autoimmune uveitis and vitreoretinal degeneration caused by mutations in the CAPN5 gene. ${ }^{1,2}$ This specific condition is rare, represented by two large American pedigrees, although there are anecdotal reports of patients that phenocopy the disease (personal communications 2012-2013). ADNIV can be divided into five disease stages. ${ }^{1}$ The first three stages are characterized by inflammatory cells in the vitreous and anterior chamber, photoreceptor degeneration, epiretinal membranes, and proliferative retinal neovascularization. Vision loss is severe in Stage IV when chronic posterior uveitis and proliferative vitreoretinopathy cause tractional retinal detachments at the macula and vitreous base (Figure 1). All patients become blind in stage V as the eyes become phthisical. ${ }^{1}$ The shared genetic origin of disease among ADNIV subjects presents a unique opportunity to study surgery in cases of posterior uveitis.

Since ADNIV patients respond poorly to conventional oral immunosuppressive medications, ${ }^{3}$ we previously studied the fluocinolone acetonide (FA) implant (Retisert ${ }^{\mathrm{TM}}$, Bausch and Lomb, Rochester, NY, USA) in ADNIV eyes. ${ }^{4}$ This device provides continuous release of intraocular corticosteroid for approximately 2.5 years 5 and demonstrated effective control of intraocular inflammation in multicentered clinical trials of noninfectious posterior uveitis. ${ }^{6}$ Intravitreal steroids may also limit retinal neovascularization and proliferative vitreoretinopathy in some reports. ${ }^{7-12}$ 


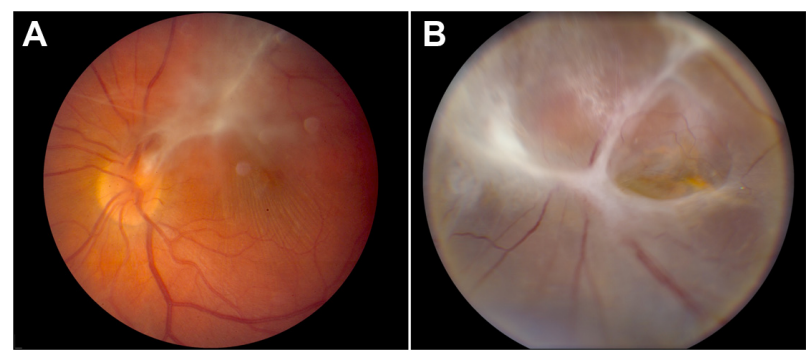

Figure I ADNIV disease stages. (A) Stage III ADNIV shows epiretinal membrane and tractional edema. (B) Stage IV shows tractional retinal detachment.

Abbreviation: ADNIV, autosomal dominant neovascular inflammatory vitreoretinopathy.

In ADINV eyes, we found that the FA implant inhibited neovascularization, inflammatory cells, and retinal edema.

Implantation of biomaterials can initiate fibrotic responses that may lead to failure of medical implants. ${ }^{13,14}$ The FA implant consists of a tablet encased in a silicone elastomer cup containing a release orifice and a polyvinyl alcohol membrane positioned between the tablet and the orifice. The silicone elastomer cup assembly is attached to a polyvinyl alcohol suture tab with silicone adhesive. The inactive ingredients include microcrystalline cellulose, polyvinyl alcohol, and magnesium stearate. Failure of the FA device due to fibrosis has not been reported in several large clinical trials and case series. ${ }^{5,15-17} \mathrm{In}$ this study, we describe two cases of fibrotic encapsulation of the FA implant and our surgical management.

\section{Methods}

The study was approved by the Institutional Review Board for Human Subjects Research and adhered to the tenets of the Declaration of Helsinki. A retrospective case series was assembled from the charts of ADNIV patients from The University of Iowa. Clinical exams for preoperative and postoperative examinations were performed by vitreoretinal specialists (authors). Genetic testing was performed as previously described. ${ }^{1}$ Surgical images were obtained using the Zeiss microscope model F88 (Carl Zeiss Surgical, Inc, Thornwood, NY, USA) and the Panasonic 6P-US522HA CCD video camera (Panasonic, Secaucus, NJ, USA).

\section{Cases and surgical technique}

A video illustrates our surgical technique to excise membranes encapsulating the steroid implant and restore its function.

\section{Patient A}

Patient A was a 31-year-old female whose father was diagnosed with ADNIV (Figure 2). The family had a c.731T $>$ C mutation in the CAPN5 gene. She had undergone cataract extraction and intraocular lens placement in both eyes at age 24 right eye
(OD), and age 25 left eye (OS). She had also undergone pars plana vitrectomy for epiretinal membranes once $\mathrm{OD}$, and twice OS, with the most recent being 1 year prior to presentation. Her ocular inflammation was being managed with monthly injections of subtenon's triamcinolone acetonide for the 5 months preceding her presentation. Her visual acuity was 20/50 OD and 20/500 OS. Her anterior segment exam showed 1+ flare and posterior chamber intraocular lenses both eyes (OU). There were epiretinal membranes and cystoid macular edema, OS more than OD. There was peripheral scatter laser OU, and a chronic, localized tractional retinal detachment nasal to the optic nerve OS. These findings were consistent with stage III ADNIV OD and stage IV ADNIV OS. She elected to undergo vitrectomy and FA implantation OS. In the two-week interval between that visit and surgery, a cellular inflammatory membrane formed over the pupil, which was removed at the time of surgery. Membranes over the macula were peeled and an FA implant was placed per manufacturer recommendations. During her postoperative course, her subjective vision improved and there was less cystoid macular edema (CME). However, she continued to have significant vitreous flare. Within 2.5 months following surgery, the anterior chamber was twice the normal depth, the FA implant could not be visualized due to pupillary and anterior vitreous membranes, and prominent membranes over the macula were noted to recur. Echography suggested proliferative anterior membranes had incorporated the intraocular lens and FA implant.

A 23-guage vitrectomy was performed from the temporal position to better access the anterior pathology. ${ }^{18}$ With scleral depression, the vitreous along the ciliary body and over the implant was debulked. Recurrent macular membranes were peeled using 23-gauge asymmetrical forceps. There was a dense fibrous capsule surrounding the implant. A 23-gauge microvitreoretinal (MVR) blade was used to carefully incise the fibrotic capsule along the edge of the strut (Figure 3) with great care taken to avoid the polyvinyl alcohol membrane and silicone elastomer cup. Next, forceps were used to peel the fibrous capsule away from the implant and towards the ciliary body. Remaining fibrous remnants were trimmed with the vitreous cutter. This dissection avoided damage to the implant and restored exposure of the drug capsule. Her acuity remained 20/500, but there was a subjective improvement in vision, and the membranes did not recur over the implant.

\section{Patient B}

Patient B was a 54-year-old female whose mother was diagnosed with ADNIV (Figure 2). The family had a c. $728 \mathrm{G}>\mathrm{T}$ mutation in the CAPN5 gene. She developed stage $\mathrm{V}$ (end stage) ADNIV with phthisis and a visual acuity 

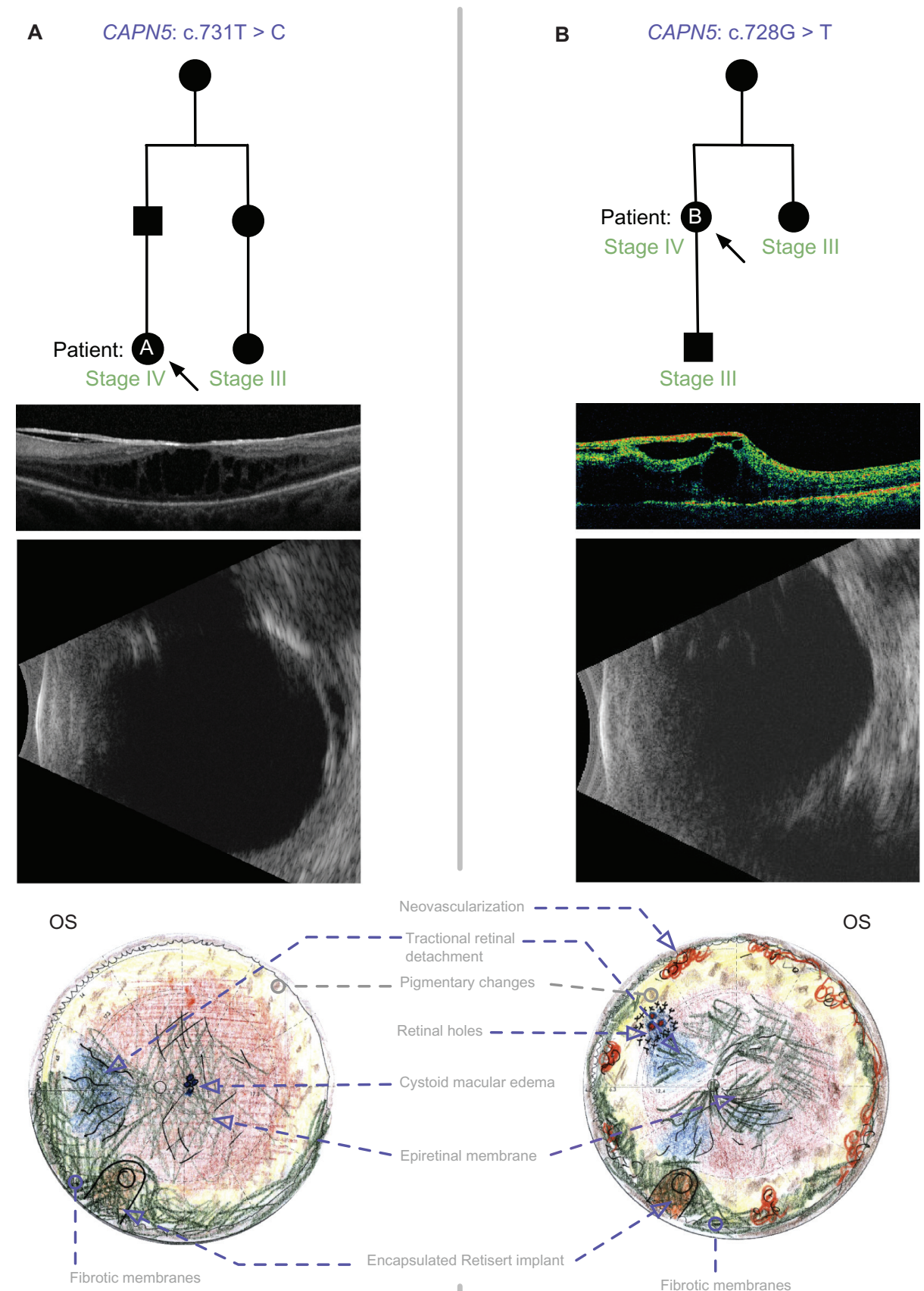

Patient A
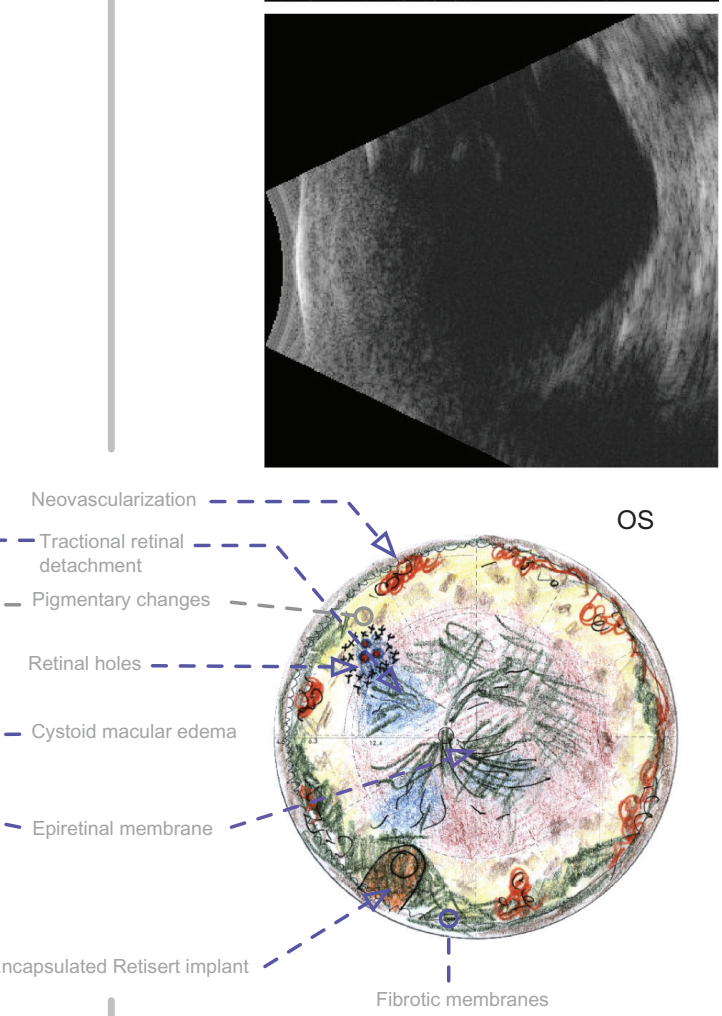

Patient B

Figure 2 ADNIV pedigree and Clinical Images. (A) Patient A. CAPN5 genotype and family pedigree; representative OCT demonstrating a thick ERM and CME in the macula; and echography reveals a peripheral TRD in Stage IV disease; the fundus drawing shows inferior, anterior PVR membranes encapsulating the implant (Retisert ${ }^{\mathrm{TM}}$, Bausch \& Lomb, Rochester, NY, USA). (B) Patient B. CAPN5 genotype and family pedigree; representative OCT showing a thick ERM and tractional elevation of the macula; echography suggests early phthisis; and the fundus drawing shows anterior neovascularization and inferior PVR membranes encapsulating the implant. In the pedigrees, arrows designate the patient with Stage IV disease and fibrotic encapsulation of the FA implant. Black symbols represent clinically affected subjects, and those that are labeled as Stage III have undergone FA implant without subsequent fibrotic encapsulation.

Abbreviations: ADNIV, autosomal dominant neovascular inflammatory vitreoretinopathy; CME, cystoid macular edema; ERM, epiretinal membrane; PVR, proliferative vitreoretinopathy; FA, fluocinolone acetonide; OCT, optical coherence tomography; OD, right eye; OS, left eye; TRD, tractional retinal detachment.

of no light perception OD following a remote repair of a tractional retinal detachment in that eye. Her visual acuity OS prior to FA implant placement was hand motion with an intraocular pressure of $20 \mathrm{mmHg}$. The anterior segment was quiet, with a posterior chamber intraocular lens in place. There was a dense, nonclearing vitreous hemorrhage with posterior vitreous bands on echography and tractional retinal detachments nasally and inferiorly, consistent with stage IV ADNIV. The patient was taking azathioprine and prednisone at this time after previously failing other 

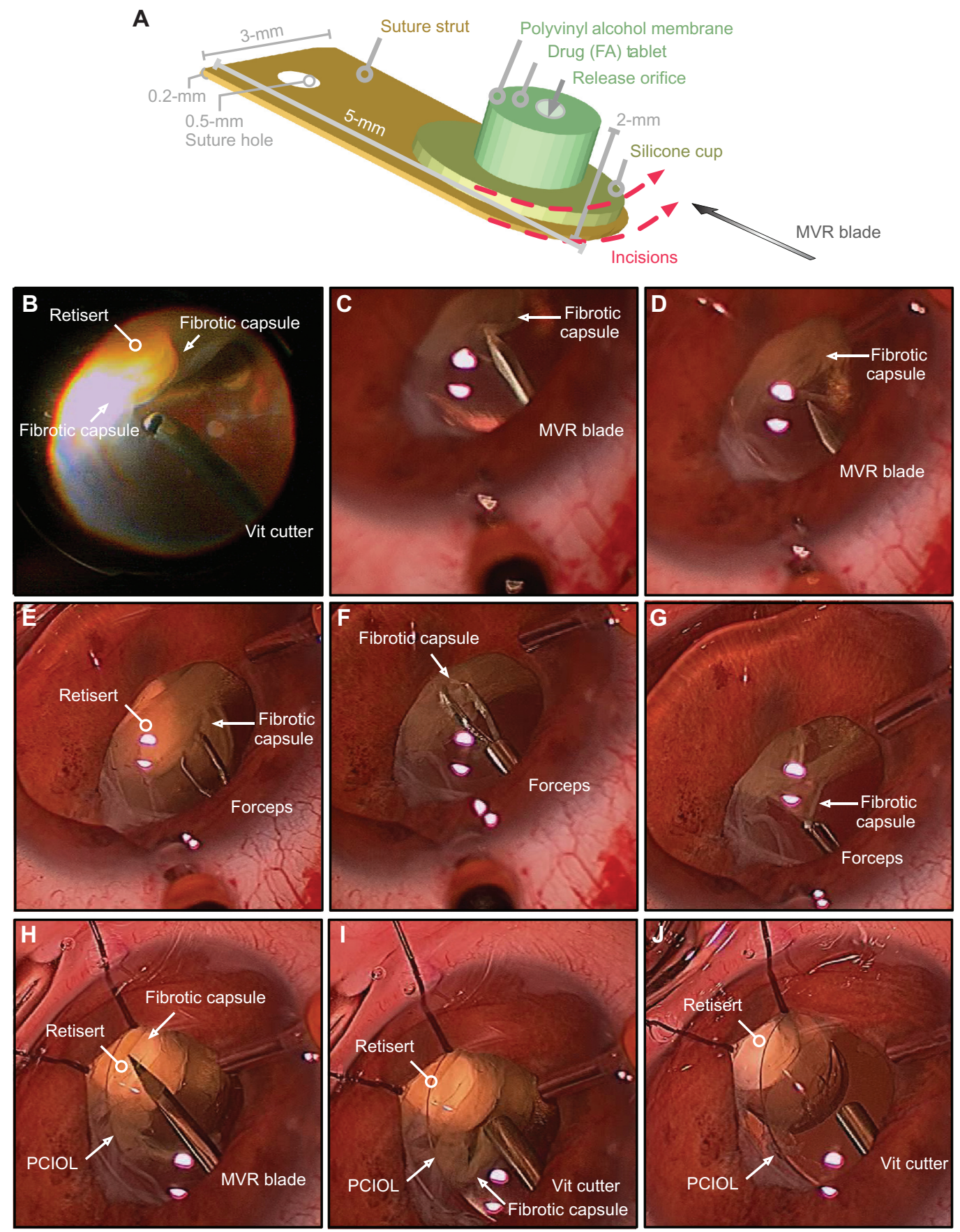

Figure 3 Surgical dissection in patient-A of fibrotic encapsulation of fluocinolone acetonide implant (Retisert ${ }^{\mathrm{TM}}$, Bausch \& Lomb, Rochester, NY, USA). (A) Diagram detailing the dimensions of the fluocinolone acetonide implant. The dotted red lines indicate the placement and orientation of incisions created in the surrounding fibrotic capsule with a microvitreoretinal (MVR) blade. (B) The vitreous cutter was used to debulk any residual vitreous surrounding the fibrotic capsule. (C and D) The MVR blade was used to incise the fibrotic capsule, taking care to avoid contact with the delicate membrane of the implant. (E-G) Intraocular forceps were used to grasp the cut edge of the membrane and peel the membrane from the surface of the implant. (H) The MVR blade was used once more to ensure that all surrounding membranes were incised. (I, J) The vitreous cutter was used to trim and excise any residual capsule, revealing the drug capsule.

Abbreviations: FA, fluocinolone acetonide; PCIL,posterior chamber intraocular lens; Vit, vitreous.

immunosuppressive agents. Pars plana vitrectomy and scleral buckling was performed with extensive membrane peeling, which revealed a retinal tear at the superior vascular arcade. An FA implant was placed in the inferonasal quadrant and a complete fluid-gas exchange was completed with $16 \%$
Octafluoropropane (C3F8) gas. Immediately following surgery, there was an aggressive posterior fibrinous response that progressed at 1 month postoperatively and did not respond to additional intravitreal triamcinolone acetonide (Kenalog, Bristol-Myers Squibb Company, Princeton, NJ, 
USA). There was reformation of pars plana bands inferiorly and subsequent hypotony. Echography also suggested the FA implant was enclosed in a fibrotic capsule.

The decision was made to proceed with a repeat vitrectomy, which was performed 4 months following the implant surgery. Thick tissue and bands were noted on the retinal surface, covering much of the pars plana and far anterior retina. There was extensive fibrin and scar formation involving most of the anterior structures, including the FA implant and intraocular lens. After the intraocular lens implant was explanted, a 20-guage vitrectomy cutter was used to debulk fibrotic vitreous overlying the FA implant and the surrounding ciliary body. Additional membranes were elevated with an intraocular pick and peeled with intraocular forceps. An inferior iridectomy was performed. Three small retinal holes in the superonasal quadrant were identified and repaired with a laser following an air-fluid exchange. The FA implant was left in place and silicone oil was reinjected. Again, within 1 month, membranes began forming over the retina. Within 2 months, the eye was hypotonous and the visual acuity in the eye became no light perception due to recurrent retinal detachment rather than device failure.

\section{Discussion}

In our experience, any intraocular surgery triggers severe inflammation in ADNIV eyes. In the preproliferative stages of ADNIV, we have used perioperative steroids to mitigate the inflammatory response. Since the fluocinolone acetonide implant is an effective device for long-term control of intraocular inflammation, we have inserted implants in nine eyes (five patients) in earlier stages of ADNIV (II-III), without fibrotic encapsulation of the device. We did, however, observe continued progression of intraocular fibrosis, ${ }^{4}$ but none of these eyes developed fibrosis over the implant. Proliferative retinal membranes characterize stage IV of ADNIV, and in the two eyes (two patients) at this stage, we observed a very aggressive fibrotic response directly at the immunosuppressive device and throughout the eye. The phenotype was similar in both patients, even though they represented two different $C A P N 5$ mutations. This suggests the molecular signals of $C A P N 5$-associated proliferative vitreoretinopathy cannot be inhibited by corticosteroids, although the dynamics of drug delivery were likely altered in the silicone-filled eye of Patient B. The fibrotic response may operate through a biological pathway similar to conventional proliferative vitreoretinopathy, which also shows a limited response to corticosteroids. ${ }^{12,19}$
The vitreous can mount a very rapid fibrotic response to encapsulate metallic foreign bodies, for example, but the material composition of the FA implant is inert. Published studies on the FA device include at least 782 implanted eyes without any report of fibrotic encapsulation, ${ }^{5,6,15-17,20-23}$ and the manufacturer has not had any such reports (personal communication, 2013). There was one report of the formation of visually significant vitreous bands in four eyes after FA implantation requiring further surgery, ${ }^{20}$ and another report detailing two cases of posterior synechia. ${ }^{24}$ It was our impression that encapsulation prevented drug delivery to the posterior segment and surgical excision of the membrane restored device function. Fibrotic encapsulation of surgical devices can lead to device failure. This has been observed with glaucoma implants ${ }^{25}$ and a variety of devices for the brain, ${ }^{26}$ inner ear, ${ }^{27}$ and knee, ${ }^{28}$ for example. Steroid application is thought to reduce fibrosis, but may be insufficient in some cases. ${ }^{14,29-31}$

Our experience suggests that implantation during earlier stages of ADNIV might show greater benefit, especially before the occurrence of intraocular fibrosis that is characteristic of Stage IV disease. Indeed, the son of Patient B was diagnosed with ADNIV and received FA implants in each eye at Stage III and did not develop encapsulation in either eye (Figure 2). Furthermore, of all eyes in our experience implanted with the FA implant placed at Stage III or earlier, none were observed to develop device encapsulation. Nevertheless, the FA implant may still be beneficial in late stages of uveitis with limited vision potential. In ADNIV patients, the FA implant prevented neovascularization, inhibited inflammatory cells, reduced the frequent steroid injections, and reduced cystoid macular edema. This may alter the course of ADNIV into a more common retinal degeneration phenotype that retains central vision despite peripheral field loss.

\section{Conclusion}

Fibrotic encapsulation of the fluocinolone acetonide implant limits drug availability, but implant function can be restored by careful vitrectomy and membrane peeling.

\section{Acknowledgments}

Financial Support: The authors are supported by National Institutes of Health Grants K08EY020530 (VBM) and Research to Prevent Blindness (New York, NY, USA).

\section{Disclosure}

VBM is a consultant to Baush \& Lomb. The authors report no other conflicts of interest in this work. 


\section{References}

1. Mahajan VB, Skeie JM, Bassuk AG, et al. Calpain-5 mutations cause autoimmune uveitis, retinal neovascularization, and photoreceptor degeneration. PLoS Genet. 2012;8(10):e1003001.

2. Bennett SR, Folk JC, Kimura AE, Russell SR, Stone EM, Raphtis EM. Autosomal dominant neovascular inflammatory vitreoretinopathy. Ophthalmology. 1990;97(9):1125-1135.

3. Mahajan VB, Vallone JG, Lin JH, et al. T-cell infiltration in autosomal dominant neovascular inflammatory vitreoretinopathy. Molecular Vision. 2010;16:1034-1040.

4. Tlucek PS, Folk JC, Orien JA, Stone EM, Mahajan VB. Inhibition of neovascularization but not fibrosis with the fluocinolone acetonide implant in autosomal dominant neovascular inflammatory vitreoretinopathy. Arch Ophthalmol. 2012;130(11):1395-1401.

5. Jaffe GJ, McCallum RM, Branchaud B, Skalak C, Butuner Z, Ashton P. Long-term follow-up results of a pilot trial of a fluocinolone acetonide implant to treat posterior uveitis. Ophthalmology. 2005;112(7):1192-1198.

6. Callanan DG, Jaffe GJ, Martin DF, Pearson PA, Comstock TL. Treatment of posterior uveitis with a fluocinolone acetonide implant: three-year clinical trial results. Arc Ophthalmol. 2008;126(9):1191-1201.

7. Bandello F, Polito A, Pognuz DR, Monaco P, Dimastrogiovanni A, Paissios J. Triamcinolone as adjunctive treatment to laser panretinal photocoagulation for proliferative diabetic retinopathy. Arch Ophthalmol. 2006;124(5):643-650.

8. Chan CK, Ip MS, Vanveldhuisen PC, et al; SCORE Study report \#11: incidences of neovascular events in eyes with retinal vein occlusion. Ophthalmology. 2011;118(7):1364-1372.

9. Jonas JB, Hayler JK, Sofker A, Panda-Jonas S. Intravitreal injection of crystalline cortisone as adjunctive treatment of proliferative diabetic retinopathy. Am J Ophthalmol. 2001;131(4):468-471.

10. Munir WM, Pulido JS, Sharma MC, Buerk BM. Intravitreal triamcinolone for treatment of complicated proliferative diabetic retinopathy and proliferative vitreoretinopathy. Can J Ophthalmol. 2005;40(5):598-604.

11. Chen W, Chen H, Hou P, Fok A, Hu Y, Lam DS. Midterm results of low-dose intravitreal triamcinolone as adjunctive treatment for proliferative vitreoretinopathy. Retina. 2011;31(6):1137-1142.

12. Ahmadieh H, Feghhi M, Tabatabaei H, Shoeibi N, Ramezani A, Mohebbi MR. Triamcinolone acetonide in silicone-filled eyes as adjunctive treatment for proliferative vitreoretinopathy: a randomized clinical trial. Ophthalmology. 2008;115(11):1938-1943.

13. Tang L, Eaton JW. Inflammatory responses to biomaterials. Am J Clin Pathol. 1995;103(4):466-471.

14. Tang L, Eaton JW. Natural responses to unnatural materials: A molecular mechanism for foreign body reactions. Mol Med. 1999;5(6):351-358.

15. Mahajan VB, Gehrs KM, Goldstein DA, Fischer DH, Lopez JS, Folk JC. Management of sympathetic ophthalmia with the fluocinolone acetonide implant. Ophthalmology. 2009;116(3):552-557.

16. Pearson PA, Comstock TL, Ip M, et al. Fluocinolone acetonide intravitreal implant for diabetic macular edema: a 3-year multicenter, randomized, controlled clinical trial. Ophthalmology. 2011;118(8):1580-1587.
17. Patel CC, Mandava N, Oliver SC, Braverman R, Quiroz-Mercado H, Olson JL. Treatment of intractable posterior uveitis in pediatric patients with the fluocinolone acetonide intravitreal implant (Retisert). Retina. 2012;32(3):537-542.

18. Tarantola RM, Graff JM, Somani R, Mahajan VB. Temporal approach for small-gauge pars plana vitrectomy combined with anterior segment surgery. Retina. 2012;32(8):1614-1623.

19. Dehghan MH, Ahmadieh H, Soheilian M, et al. Effect of oral prednisolone on visual outcomes and complications after scleral buckling. Eur J Ophthalmol. 2010;20(2):419-423.

20. Galor A, Margolis R, Kaiser PK, Lowder CY. Vitreous band formation and the sustained-release, intravitreal fluocinolone (Retisert) implant. Arch Ophthalmol. 2007;125(6):836-838.

21. Jain N, Stinnett SS, Jaffe GJ. Prospective study of a fluocinolone acetonide implant for chronic macular edema from central retinal vein occlusion: thirty-six-month results. Ophthalmology. 2012; 119(1):132-137.

22. Multicenter Uveitis Steroid Treatment (MUST) Trial Research Group, Kempen JH, Altaweel MM, Holbrook JT, et al. Randomized comparison of systemic anti-inflammatory therapy versus fluocinolone acetonide implant for intermediate, posterior, and panuveitis: the multicenter uveitis steroid treatment trial. Ophthalmology. 2011;118(10): 1916-1926.

23. Pavesio C, Zierhut M, Bairi K, Comstock TL, Usner DW. Evaluation of an intravitreal fluocinolone acetonide implant versus standard systemic therapy in noninfectious posterior uveitis. Ophthalmology. 2010;117(3): 567-575.

24. AhmadZM, Hughes BA, Abrams GW, MahmoudTH. Combined posterior chamber intraocular lens, vitrectomy, Retisert, and pars plana tube in noninfectious uveitis. Arch Ophthalmol. 2012;130(7):908-913.

25. Molteno AC, Fucik M, Dempster AG, Bevin TH; Otago Glaucoma Surgery Outcome Study: factors controlling capsule fibrosis around Molteno implants with histopathological correlation. Ophthalmology. 2003;110(11):2198-2206.

26. PolikovVS, Tresco PA, Reichert WM. Response of brain tissue to chronically implanted neural electrodes. J Neurosci Methods. 2005; 148(1):1-18.

27. Bohl A, Rohm HW, Ceschi P, et al. Development of a specially tailored local drug delivery system for the prevention of fibrosis after insertion of cochlear implants into the inner ear. J Mater Sci Mater Med. 2012; 23(9):2151-2162.

28. Parratte S, Boyer P, Piriou P, et al. Total knee replacement following intra-articular malunion. Orthop Traumatol Surg Res. 2011; 97(Suppl 6):S118-S123.

29. Butler KR Jr, Benghuzzi HA. Immunohistochemical detection of cytokine expression in tissue-implant response associated with TCP bioceramic implants loaded with steroid hormones. Biomed Sci Instrum. 2003;39:541-546.

30. Hickey T, Kreutzer D, Burgess DJ, Moussy F. In vivo evaluation of a dexamethasone/PLGA microsphere system designed to suppress the inflammatory tissue response to implantable medical devices. J Biomed Mater Res. 2002;61(2):180-187.

31. Patil SD, Papadimitrakopoulos F, Burgess DJ. Dexamethasone-loaded poly(lactic-co-glycolic) acid microspheres/poly(vinyl alcohol) hydrogel composite coatings for inflammation control. Diabetes Technol Ther. 2004;6(6):887-897.
Clinical Ophthalmology

\section{Publish your work in this journal}

Clinical Ophthalmology is an international, peer-reviewed journal covering all subspecialties within ophthalmology. Key topics include: Optometry; Visual science; Pharmacology and drug therapy in eye diseases; Basic Sciences; Primary and Secondary eye care; Patient Safety and Quality of Care Improvements. This journal is indexed on Submit your manuscript here: http://www.dovepress.com/clinical-ophthalmology-journal

\section{Dovepress}

PubMed Central and CAS, and is the official journal of The Society of Clinical Ophthalmology (SCO). The manuscript management system is completely online and includes a very quick and fair peer-review system, which is all easy to use. Visit http://www.dovepress.com/ testimonials.php to read real quotes from published authors. 